\title{
UMA EXTENSÃO DO MOODLE PARA RECOMENDAÇÃO UBÍQUA DE OBJETOS DE APRENDIZAGEM
}

\author{
Luiz Jácome Júnior ${ }^{1}$ \\ Francisco Milton Mendes Neto ${ }^{1}$ \\ Cecília Dias Flores ${ }^{2}$ \\ Luiz Cláudio Nogueira da Silva ${ }^{3}$ \\ Enio Lopes Sombra ${ }^{3}$ \\ Alisson Alan Lima da Costa ${ }^{3}$

\begin{abstract}
${ }^{1}$ Programa de Pós-Graduação em Ciência da Computação - PPGCC, Departamento de Saúde de Porto Alegre - UFCSPA.

${ }^{3}$ Superintendência de Tecnologia da Informação e Comunicação - SUTIC Universidade
\end{abstract} \\ Ciências Exatas e Naturais - DCEN, Universidade Federal Rural do Semi-Árido - UFERSA. \\ 2Programa de Pós-Graduação em Ciências da Saúde da Universidade Federal de Ciências da \\ Federal Rural do Semi-Árido - UFERSA. \\ luizjunior05@gmail.com; miltonmendes@ufersa.edu.br; dflores@inf.ufrgs.br; \\ luizclaudio@ufersa.edu.br; \{eniosombra, alisson.lyma\}@gmail.com
}

\begin{abstract}
Resumo. Paralelo ao desenvolvimento e popularização dos recursos tecnológicos, surgiu a preocupação, por parte dos educadores, de buscar meios de adotar novas tecnologias no âmbito educacional. Atualmente estão disponíveis vários ambientes para suporte à aprendizagem a distância, dentre os quais encontra-se o Moodle. O Moodle é um sistema de gestão de aprendizagem muito popular entre educadores de todo o mundo. No entanto, apesar de já possuir suporte a aprendizagem móvel, ainda não possui um meio que contemple os benefícios oferecidos pela aprendizagem ubíqua. Diante deste cenário, o presente artigo propõe o desenvolvimento de uma extensão para o Moodle capaz de fornecer um ambiente de aprendizagem ubíqua, por meio da recomendação de objetos de aprendizagem sensível ao contexto do estudante.
\end{abstract}

Palavras-chaves: sistemas de recomendação, objetos de aprendizagem, aprendizagem ubíqua.

\section{AN EXTENSION OF MOODLE FOR UBIQUITOUS RECOMMENDATION OF LEARNING OBJECTS}

\begin{abstract}
Parallel to the development and popularization of technological resources, emerged a concern, on the part of educators, of seek ways to adopt new technologies in education. Currently, several environments to support distance learning are available, among them is the Moodle. Moodle is a learning management system very popular among educators around the world. However, despite already owning mobile learning support, it has not a way that contemplates the benefits offered by ubiquitous learning. Given this scenario, this paper proposes the development of an extension to Moodle able to provide an ubiquitous learning environment, through the recommendation of context-aware learning objects.
\end{abstract}

Keywords: recommendation systems, learning objects, ubiquitous learning. 


\section{Introdução}

O desenvolvimento tecnológico impulsionou o surgimento de novas possibilidades educacionais, bem como o aparecimento de ferramentas de suporte aos processos de ensino/aprendizagem. O Moodle é um pacote de software para produzir web sites e cursos baseados na web, voltados ao âmbito educacional. Trata-se de um Sistema de Gestão de Aprendizagem (Learning Management System - LMS) muito popular entre educadores de todo o mundo (Moodle, 2012).

A aprendizagem móvel (do inglês Mobile Learning ou m-Learning) permite que a aprendizagem ocorra a qualquer hora e em qualquer lugar. Porém, apesar de prover mobilidade, ela não fornece uma aprendizagem capaz de considerar informações sensíveis ao contexto do estudante (Mandula et al, 2011). Ao considerarmos as características da aprendizagem móvel e a capacidade de detectar as informações de contexto do estudante, a fim de fornecer conteúdo educacional de forma personalizada, surge o conceito de Aprendizagem Ubíqua (também conhecida como u-learning, de ubiquitous learning) (Saccol et al, 2010).

Utilizando o Moodle, é possível elaborar e aplicar cursos voltados para $\mathrm{m}$ learning através da utilização do plugin MLE-Moodle. Este plugin permite que a aprendizagem a distância suportada por computador, conhecida por e-learning, seja oferecida por meio de dispositivos móveis. No entanto, o Moodle não possui um meio que contemple os benefícios didático-pedagógicos oferecidos pela u-learning (MLE, 2012).

No cenário atual, onde os estudantes possuem acesso a uma vasta variedade e quantidade de conteúdo educativo, através de dispositivos portáteis, a u-learning possui um papel fundamental para o processo de aprendizagem no qual não existe a presença física de um educador. Logo, utilizando-se de um sistema de recomendação, é possível direcionar o estudante de forma a maximizar seu aprendizado (Cazella et al, 2011). Entretanto, é essencial a padronização dos conteúdos educacionais para que seja possível a automatização eficiente do processo de recomendação de conteúdo adequado ao contexto do estudante. Um modo eficaz de padronizar conteúdos educacionais é através do uso de Objetos de Aprendizagem (OAs), os quais consistem em pequenas unidades de conteúdo que podem ser usadas, reutilizadas e referenciadas durante um processo de aprendizagem (LTSC, 2002).

Este artigo apresenta uma extensão ao Moodle que possibilita fornecer um ambiente de aprendizagem ubíqua. A extensão proposta utiliza técnicas de inteligência artificial, no caso agentes inteligentes e Algoritmo Genético (AG), para desenvolver um Sistema de Recomendação Híbrido (Martins et al, 2011) capaz de fornecer conteúdos educacionais sensíveis às características particulares de cada estudante, como também atender as restrições dos seus dispositivos móveis, uma vez que os mesmos possuem recursos distintos e limitados. Desta forma, essa extensão pretende integrar, de maneira apropriada, um sistema de recomendação ao MLE-Moodle, a fim de fornecer um ambiente de aprendizagem móvel sensível ao contexto do estudante, caracterizando a aprendizagem ubíqua.

Este artigo está dividido em sete seções. A Seção 2 apresenta o conceito de aprendizagem ubíqua. A Seção 3 discute o conceito de sistema de recomendação. A Seção 4 discute os trabalhos relacionados. A Seção 5 descreve a extensão para o Moodle proposta neste artigo. A Seção 6 demonstra um cenário de uso do sistema 
proposto. A última seção apresenta nossas considerações finais e uma discussão sobre trabalhos futuros.

\section{Aprendizagem Ubíqua}

Mark Weiser (1991) idealizou o mundo da computação ubíqua, onde o computador deixa de ser usado como um dispositivo de propósito geral, passando a ter uma nova visão de sua funcionalidade, atendendo as necessidades específicas de cada usuário. Neste cenário, um usuário não se restringe a acessar um único computador, mas vários dispositivos computacionais interligados entre si e disseminados de forma quase imperceptível pelo ambiente.

A partir do conceito apresentado pode-se definir a aprendizagem ubíqua como sendo a utilização de dispositivos móveis, tecnologias de comunicação móvel sem fio, sensores e mecanismo de localização, com o objetivo de auxiliar o processo educacional, levando em consideração características particulares dos estudantes (Saccol et al, 2010).

Desta forma, a aprendizagem ubíqua pode ser considerada como sendo a aprendizagem móvel que é realizada levando-se em consideração as características do contexto dos estudantes, provendo a estes conteúdos adaptados às suas necessidades (Mandula et al, 2011). Assim, a aprendizagem ubíqua provê um novo paradigma, através do uso de dispositivos móveis, que fornece um serviço de forma transparente aos estudantes (Loureiro et al, 2009).

A mobilidade permitida ao usuário devido ao uso de dispositivos móveis em aplicações ubíquas torna ainda mais importante a consideração do contexto de um estudante, visto que as características do estudante podem se modificar a qualquer momento. Essas modificações podem ocorrer em diversos aspectos (ex. condições físicas, recursos físicos disponíveis, recursos computacionais etc.) (Loureiro et al, 2009).

\section{Sistemas de Recomendação de OAs}

De acordo com Schafe (1999) citado por (Vieira e Nunes, 2012), o aumento de meios de disponibilização de conteúdo, podendo ser produtos ou informação, através de sistemas Web, provoca uma situação onde o usuário possui muitas opções de escolha antes mesmo de estar apto a selecionar uma opção que atenda suas necessidades. Os Sistemas de Recomendação (SR) buscam amenizar os impactos gerados por essa sobrecarga de informação.

Os SRs utilizam repositórios de informação e dados de preferência dos usuários para direcionar conteúdos aos indivíduos com potenciais interesses. Um dos desafios dos SRs é realizar a indicação de produtos, serviços e/ou informação que melhor atendam as expectativas dos usuários (e ao seu perfil) (Cazella et al, 2011).

Segundo Vieira e Nunes (2012), os SRs podem ser classificados em três tipos: Sistema de Recomendação Baseada em Conteúdo (SRBC), Sistema de Recomendação Colaborativa (SRC) e Sistema de Recomendação Híbrida (SRH). A recomendação baseada em conteúdo é realiza através de informações recuperadas do usuário (perfil, comportamento) e/ou do seu histórico de escolhas. Já a recomendação por filtragem colaborativa leva em consideração escolhas realizadas por usuários com características 
similares. Por último, o SRH efetua a recomendação através da aplicação das técnicas utilizadas pelos SRBC e SRC, de forma a atingir um maior número de possibilidades e sugerir objetos que diretamente não aparentam estar relacionados (Martins, 2011).

No âmbito da educação ubíqua, os alunos são expostos a uma grande quantidade de OAs e podem levar um bom tempo para realizar escolhas difíceis. Tendo em vista que os estudantes estão em processo de formação, estes não estão aptos o suficiente para realizar tais escolhas. Um SR vem a suprir esta carência recomendando OAs apropriados e tirando a responsabilidade de escolha do estudante.

\section{Trabalhos relacionados}

A adaptação de ambientes virtuais de aprendizagem às características particulares dos estudantes já é um tema bastante abordado em pesquisas acadêmicas. Rani, Ashok e Palanivel (2009) apresentam uma abordagem para personalização de aprendizagem eletrônica (ou electronic learning ou ainda e-learning) com base em ontologia, na qual a troca de informações é mantida por serviços Web (web services), sob uma arquitetura orientada a serviços. A ideia principal do sistema é identificar requisitos do usuário, ou seja, as preferências dos estudantes e suas características, e criar o modelo do estudante. Nesse modelo constará o seu conhecimento expresso através de um conjunto de palavras-chave pertencentes a uma ontologia comum para, de acordo com o modelo de cada estudante, adaptar o curso de forma individual.

Gluz e Vicari (2010) propuseram a MILOS (Multiagent Infrastructure for Learning Object Support), uma infraestrutura, combinando ontologias e agentes, que implementa as funcionalidades necessárias aos processos de autoria, gerência, busca e disponibilização de OAs compatíveis com a proposta de padrão de metadados de OAs OBAA (Objetos de Aprendizagem Baseados em Agentes).

Já Ferreira e Raabe (2010) apresentam o LorSys (Learning Object Recommender System), um sistema de recomendação de OAs, no formato SCORM, para o Moodle. O LorSys utiliza a técnica de recomendação híbrida, aplicada à informações de preferências dos estudantes e dados de acesso aos OAs. O estudante visualiza os OAs recomendados por meio de um bloco (componente do Moodle) adicionado na interface dos cursos.

O presente trabalho aprimora o Moodle por meio do desenvolvimento de um serviço de recomendação de OAs que, além de atender o perfil de aprendizagem de cada aluno, considera os recursos dos dispositivos móveis durante o processo de recomendação. Foram utilizadas técnicas de inteligência artificial para o desenvolvimento do SRH. O SRH é disponibilizado como Serviço Web (Web Service) e sua integração com o Moodle é realizada através de um plugin desenvolvido em conformidade com os padrões estabelecidos pelo LMS. Portanto, este trabalho provê um meio para que o Moodle seja capaz fornecer um ambiente de aprendizagem ubíqua.

\section{Uma Extensão do Moodle para Recomendação Ubíqua de Objetos de Aprendizagem}

A maneira apropriada de contribuir para o desenvolvimento do Moodle é por meio da criação de plugins, seguindo a especificação fornecida pelo LMS (Moodle Developer Documentation, 2012). Uma vez desenvolvido um plugin, é possível disponibilizá-lo para que seja utilizado por outros administradores desse LMS. A extensão para o 
Moodle proposta neste trabalho busca integrar, de maneira adequada, um sistema de recomendação, elaborado a partir de técnicas de inteligência artificial, com o MLEMoodle.
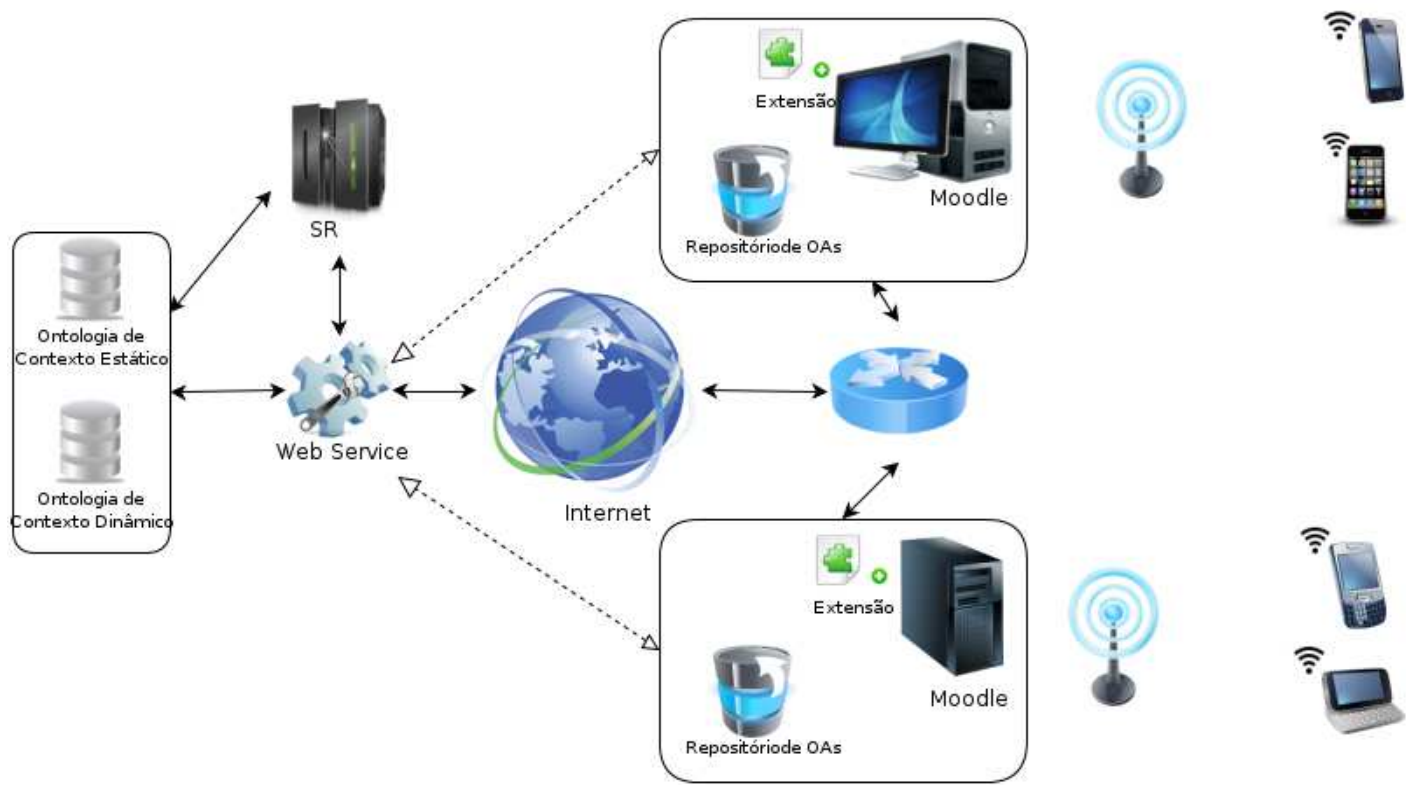

Figura 1 - Arquitetura do Sistema
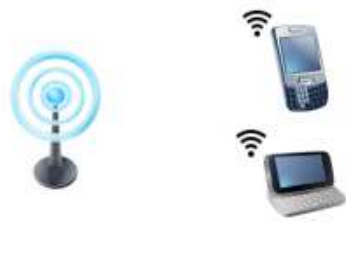

Como apresentado na Figura 1, a extensão incorporada ao Moodle fornece um meio de comunicação com um Serviço Web, do inglês Web Service (Sommerville, 2011), responsável por disponibilizar o serviço de recomendação. O Serviço Web foi desenvolvido para facilitar a integração e padronizar a comunicação do SR com a plataforma do Moodle. Deste modo, a extensão envia as informações necessárias para o serviço de recomendação e recebe os resultados do processo, ou seja, os OAs recomendados.

\subsection{Descrição do Serviço Web}

Serviço Web é uma das soluções mais utilizadas para integração de sistemas e na comunicação entre aplicações diferentes. Esta tecnologia permite a compatibilidade de softwares desenvolvidos em diferentes plataformas. Desta forma, os Serviços Web fornecem uma solução que define um padrão para troca de mensagens entre as aplicações consumidoras (clientes) e um fornecedor do serviço.

O desenvolvimento do Serviço Web proposto para extensão do Moodle utilizou a arquitetura RESTful (RESTful, 2012). Trata-se de uma arquitetura de transferência de estado que fornece serviços de acesso a recursos. As informações da aplicação e de transações são estruturadas nos formatos XML (eXtensible Markup Language) (XML, 2012), JSON (JSON, 2012), entre outros. Esta arquitetura possui um pequeno conjunto de operações para manipulação das informações dos recursos. Os componentes da rede se comunicam através do protocolo HTTP (Hypertext Transfer Protocol) (HTTP, 2012), com o objetivo de trocar representações de recursos.

O Java EE (Enterprise Edition) (Java EE, 2012) integra o JSR-311 (Java Specification Requests) (RESTful, 2012) para fornecer um conjunto de especificações que define a criação de um Serviço Web com a arquitetura RESTful. Para a 
implementação de referência do JSR-311, utilizou-se o Jersey (Jersey, 2012), que é um projeto open source mantido pela Oracle (Oracle, 2012).

O Serviço Web desenvolvido tem como objetivo fornecer o serviço de recomendação de OAs para a plataforma do Moodle. Porém, da forma como foi desenvolvido, é possível atender outros LMS realizando poucas alterações.

Para que seja possível realizar a recomendação de OAs a um estudante, é necessário, inicialmente, capturar informações do seu perfil. Para isso, ele precisa realizar um cadastro no qual irá informar algumas características de seu perfil, como, por exemplo, local e hora do dia preferido para o estudo, sua área de interesse, entre outras. Estas informações são enviadas para o Serviço Web e mantidas na ontologia de contexto estático dos estudantes.

Já as informações dinâmicas (localização geográfica, horário atual de acesso ao ambiente e identificação do dispositivo móvel utilizado) são capturadas no momento do acesso do estudante. Estas são enviadas e mantidas na ontologia de contexto dinâmico do estudante, através do Serviço Web.

O Moodle mantém um repositório local de OAs. Para que o mecanismo de recomendação funcione, é necessário que estes OAs estejam em conformidade com o padrão SCORM (ADL, 2012). Através dos metadados descritos nesse padrão, os agentes serão capazes de comparar as informações dos OAs com os perfis dos estudantes e realizar a recomendação de OAs de maneira adequada. O Serviço Web mantém uma base de dados contendo os arquivos de descrição dos OAs referentes a cada repositório dos LMS integrados. Cabe à extensão a responsabilidade de realizar atualizações nessa base de dados, por meio de chamadas ao Serviço Web.

\subsection{Sistema de Recomendação de OAs}

A seguir serão descritos o SMA e o AG que compõem o SRH. Esses componentes possibilitam automatizar o processo de recomendação de OAs sensível ao contexto do estudante.

\subsubsection{Sistema Multiagente}

O SR é formado por um SMA do tipo comunidade de especialistas, onde cada agente é especialista em uma determinada tarefa. O SMA foi desenvolvido utilizando a plataforma de desenvolvimento e execução de agentes de software conhecida como JADE (Java Agent Development Framework) (JADE, 2012). Nessa plataforma, os agentes interagem entre si através de um protocolo de comunicação previamente estabelecido.

O SMA é composto de quatro tipos de agentes: Agente Estudante (Student Agent - SAg), Agente Recomendador (Recommender Agent - RAg), Agente de Interface (Interface Agent - IAg) e Agente DF (Directory Facilitator).

O agente DF possui o comportamento voltado para a mediação da comunicação entre os outros agentes. Sua função principal é fornecer uma arquitetura do tipo "quadro-negro", onde agentes escrevem informações, procuram por informações escritas por outros agentes e conseguem, através do serviço provido pelo DF, se comunicar com o agente que escreveu aquela informação. 
Os SAgs são responsáveis por monitorar as atividades dos estudantes, atualizar e recuperar, das ontologias de contexto estático e dinâmico, as preferências de aprendizagem que compõem os perfis dos estudantes e seus respectivos históricos de escolhas de OAs. Os SAgs também capturam as informações de localização geográfica e do horário corrente. Ou seja, o comportamento do SAg é voltado para monitoramento das informações relativas ao estudante. Em seguida, todas essas informações são cadastradas no agente DF.

O RAg tem o intuito de detectar OAs adequados ao contexto do estudante, levando em consideração (i) as informações consultadas no agente DF, (ii) as informações dos OAs disponíveis no repositório de OAs SCORM e (iii) as preferências similares de outros estudantes. Após identificar os OAs adequados, o RAg cadastra, no agente DF, as novas informações, as quais servirão ao IAg.

O IAg é responsável, principalmente, por verificar a adequação visual do OA recomendado pelo RAg às características do dispositivo móvel do estudante. Caso o OA não seja adequado ao dispositivo, o IAg rejeitará a recomendação. Além disso, o IAg tem a responsabilidade de informar ao tutor o conteúdo que foi sugerido.

\subsubsection{Algoritmo Genético}

O SMA utiliza um AG para identificar os OAs a serem recomendados. A utilização do AG é justificada pela alta complexidade do problema de recomendação, o que pode depreciar o desempenho do SMA quando o número de OAs for suficientemente grande. Portanto, a aplicação de um algoritmo exato seria inviável. Neste caso, um algoritmo aproximativo (AG) viabiliza a busca por uma solução próxima da ótima.

Para realizar uma recomendação de OAs adequada ao contexto do estudante, é essencial levar em consideração características que podem limitar a aprendizagem do mesmo.

Inicialmente, temos uma população composta de certa quantidade de OAs. Cada OA possui uma série de características que o relaciona com o contexto do estudante e são consideradas durante o processamento do AG. No presente trabalho, foram consideradas as seguintes características:

i) Afinidade com o curso: representa o grau de relacionamento do conteúdo de um OA a determinado curso. Esse grau de afinidade é representado no AG em uma escala que varia de 0 (nenhuma afinidade) a 5 (alta afinidade). Determinado OA pode estar relacionado a um ou vários cursos, sendo o administrador de cada curso responsável por determinar, manualmente, quais OAs são afins ao curso e qual o seu grau de afinidade.

ii) Afinidade com o dispositivo móvel: determina o grau de adequação do conteúdo de um $\mathrm{OA}$ ao dispositivo móvel do estudante. $\mathrm{O}$ grau de afinidade com o dispositivo é definido no $\mathrm{AG}$ de acordo com uma faixa que varia de 0 (nenhum recurso suportado) a 5 (todos os recursos suportados). As informações do dispositivo são capturadas a partir da requisição HTTP enviada do dispositivo móvel para o servidor no qual se encontra o LMS. Em seguida, em posse dessas informações, é possível verificar em uma base de dados, chamada WURFL (WURFL, 2012), quais recursos aquele dispositivo móvel suporta.

iii) Horário de estudo: os horários de estudo são definidos em faixas de horários. O estudante define, inicialmente, qual o horário de estudo preferido dele. Assim, 
quando o estudante acessa o ambiente de aprendizagem, o sistema se encarrega de verificar se o horário corrente está incluso na faixa de estudo preferida do estudante. Caso esteja nessa faixa, o sistema atribui o maior valor possível a essa variável no AG (valor 5). Do contrário, será atribuído um valor cada vez menor, sendo o mínimo 1, à medida que o horário corrente se distancie daquele definido como preferido pelo estudante.

iv) Localização corrente: outra informação que pode ser levada em consideração para a definição do contexto do estudante é a localidade na qual ele se encontra. Em posse dessa informação, é possível verificar, nos metadados do padrão de OAs LOM (Learning Object Metadata) (LTSC, 2002), a incidência de palavras relacionadas à localização do estudante. Com base nisso, são capturadas e consideradas informações do país, estado e cidade na qual o estudante se encontra. Quanto mais palavras relacionadas à localização do estudante forem encontradas, maior será o valor atribuído a essa variável no AG (máximo 5). Por outro lado, caso não seja encontrada nenhuma informação relacionada, será atribuída a pontuação mínima (valor 0).

v) Escolhas de outros estudantes: define um valor proporcional que representa a quantidade de acessos a um OA. A informação de quais OAs são mais acessados é levada em consideração em conjunto com a informação de afinidade do OA com o curso, pois de nada adiantaria para a recomendação um OA ser o mais acessado e não possuir nenhuma afinidade com o curso em questão. Desta forma, esse valor pode variar de 0 a 5 dependendo da quantidade de acessos e da sua relação com o curso.

vi) Incidência de palavras-chave: os OAs disponibilizados no padrão SCORM possuem metadados que informam as palavras-chave relacionadas ao assunto daquele OA (tagkeywords), além do título deste. Essas informações estão localizadas, mais especificamente, no padrão LOM, o qual é parte integrante do padrão SCORM. Em posse dessas informações, é feita uma verificação da incidência dessas palavras na descrição do curso do qual o estudante está participando. Quanto maior a quantidade de palavras-chave relacionadas, a probabilidade de que o OA possua assuntos relacionados ao curso será maior, sendo pontuado, portanto, com um peso maior (no máximo 5). Por outro lado, quanto menor a quantidade de palavras-chave relacionadas, o peso atribuído ao OA nessa variável no AG torna-se proporcionalmente menor.

No AG, cada cromossomo é formado por um conjunto de três OAs. A representação do OA (gene) é formada pelo ID e sua respectiva aptidão. O cálculo da aptidão do OA é o somatório dos valores atribuídos a cada característica citada anteriormente. Já a aptidão do indivíduo é o somatório das aptidões dos OAs que compõem os genes.

Essa abordagem de um cromossomo contendo três OAs foi utilizada porque, no final da execução do AG, será indicado um cromossomo como o indivíduo mais apto, ou seja, como a melhor solução levando-se em consideração o contexto em questão. Portanto, não seria interessante, recomendar apenas um $\mathrm{OA}$, mas sim um conjunto dentre o qual o estudante pudesse escolher aquele que lhe fosse mais conveniente.

\section{Descrição de um Cenário de Uso}

O estudante acessa o site do Moodle e se autentica no sistema, fornecendo nome de usuário e senha. Na página do usuário ele tem a opção de responder um questionário para auxiliar na personalização do ambiente. Este questionário busca identificar as 
preferências de conteúdo, local e horário de estudo do estudante. Uma vez preenchido o questionário, o estudante é direcionado para uma página que contém uma lista de fabricantes de dispositivos móveis. Ele seleciona o fabricante do seu dispositivo móvel e, posteriormente, o link que apresenta o modelo para seu aparelho. Em seguida, ele é direcionado para uma página de download do MLE-Moodle que melhor se adapta ao seu dispositivo. $\mathrm{O}$ estudante instala o aplicativo no seu aparelho e, ao executar a aplicação, envia (de forma transparente) informações de autenticação e localização ao servidor. Depois de executar a aplicação, o estudante é direcionado para o menu principal, através do qual decide verificar se existe algum conteúdo sugerido, escolhendo a opção - Messages. Na próxima tela, o estudante percebe que existem mensagens não lidas na caixa de entrada. Dentre as mensagens não lidas ele identifica uma que tem como remetente o agente recomendador. Essa mensagem apresenta ao estudante o conteúdo sugerido de acordo com seu perfil de aprendizagem e seu contexto. Em seguida, o estudante retorna ao menu principal e acessa o menu - My courses, onde verifica informações do OA sugerido e acessa seu conteúdo.

\section{Considerações Finais e Trabalhos Futuros}

A comunidade científica tem dedicado esforços para tornar os ambientes virtuais de aprendizagem mais próximos da realidade do ensino presencial. A utilização de dispositivos móveis como meio de prover ensino, apesar da sua característica motivadora, trouxe novos desafios. Um ambiente de aprendizagem ubíqua, além de possuir as características apresentadas pela aprendizagem móvel, é capaz de adaptar-se às necessidades específicas dos estudantes. Essa adaptação pode ser explorada por meio da adequação de conteúdo educativo ao estudante de acordo com suas preferências de aprendizagem.

Os ambientes virtuais de aprendizagem, em geral, dedicam maior atenção aos aspectos funcionais, esquecendo a função didático-pedagógica. Pensando nisso, este artigo apresentou uma extensão para o LMS Moodle capaz de fornecer um ambiente de aprendizagem ubíqua, através da recomendação de objetos de aprendizagem sensível ao contexto do estudante. Desta forma, pretende-se aperfeiçoar o processo de ensinoaprendizagem de forma transparente aos estudantes.

Como trabalho futuro, pretende-se disponibilizar a extensão para validação da comunidade de desenvolvedores do Moodle. Em um segundo momento, almeja-se realizar um estudo de caso em um contexto real. Isso possibilitará mensurar, estatisticamente, o quanto este mecanismo de recomendação contribui para o processo de ensino-aprendizagem.

\section{Referências}

ADL. Advanced distributed learning, Disponível em: 〈http://www.adlnet.org>. Acessado em: 29 out. 2012.

Cazella, S. C., Silva, K. K., Bhear, P., Schneider, D. and Freitas, R. Recomendando Objetos De Aprendizagem Baseado em Competências em Ead, RENOTE - Revista Novas Tecnologias na Educação, CINTED-UFRGS, ISSN 1679-1916, v. 9, n.2, 2011.

Ferreira, V. H. and Raabe, A. L. A. LORSys - Um Sistema de Recomendação de Objetos de Aprendizagem SCORM, RENOTE - Revista Novas Tecnologias na Educação, CINTEDUFRGS, ISSN 1679-1916, v. 8, n.2, 2010. 
Gluz, J.C., Vicari, R. M. MILOS: Infraestrutura de Agentes para Suporte a Objetos de Aprendizagem OBAA, In: XXI Simpósio Brasileiro de Informática na Educação, SBIE, João Pessoa, PB, 2010.

HTTP. HyperText Transfer Protocol. Disponível em: <http://www.w3.org/Protocols/>. Acessado em: 30 Out. 2012.

JADE. Java development framework: an open-source platform for peer-to-peer agent based applications. Disponível em: <http://jade.tilab.com/>. Acessado em: 28 out. 2012.

JAVA EE. Java Enterprise Edition. Disponível em: <http://www.oracle.com/technetwork/java/javaee/overview/index.html>. Acessado em: 30 Out. 2012.

Jersey. Reference Implementation for building RESTful Web services, Disponível em: <http://jersey.java.net/>. Acessado em: 28 out. 2012.

JSON. JavaScript Object Notation. Disponível em: <http://www.json.org/>. Acessado em: 30 Out. 2012.

Loureiro, A. A. F. et al. Computação Ubíqua Ciente de Contexto: Desafios e Tendências. In: XXVII Simpósio Brasileiro de Redes de Computadores e Sistemas Distribuídos (SRBC'09). Recife, PE: SBIE. 2009.

LTSC. Draft Standard for Learning Object Metadata. IEEE Standard 1484.12.1. LTSC, Learning Technology Standards Committee, New York: Institute of Electrical and Electronics Engineers, 2002.

MLE. MLE - Mobile Learning Engine, Disponível em: <http://mle.sourceforge.net/mle/index.php>. Acessado em: 20 Abr. 2012.

Mandula, K.; Meda, S. R.; Kambham, R. Implementation of Ubiquitous Learning System Using Sensor Technologies. In: IEEE International Conference on Technology for Education. Hyderabad, India: IEEE Computer Society. p. 142-148, 2011.

Martins, H. N. J., Costa, E. B., Oliveira, T. T. M., Bittencourt, I. I. Sistema de Recomendação Híbrido para Bibliotecas Digitais que Suportam o Protocolo OAI-PMH, Artigo Completo, In: XXII Simpósio Brasileiro de Informática na Educação, SBIE, Aracaju, SE, 2011.

Moodle. Moodle - Sistema Open Source de Gerenciamento de Cursos, Disponível em: <http://moodle.org/about/>. Acessado em: 20 Mar. 2012.

Moodle Developer Documentation. Developer documentation, Disponível em: <http://docs.moodle.org/dev/Developer_documentation>. Acessado em: 20 Mar. 2012.

Oracle. Disponível em: <http://www.oracle.com/index.html>. Acessado em: 30 Out. 2012.

Rani, S. J.; Ashok, M. S.; Palanivel, K. Adaptive content for personalized E-learning using web service and semantic web. In: International Conference on Intelligent Agent Multi-Agent Systems (IAMA). Pondicherry, India: IEEE Computer Society. p. 1-4, 2009.

RESTful. RESTful Web Services. Disponível em: <http://www.oracle.com/technetwork/articles/javase/index-137171.html>. Acessado em: 20 Mar. 2012.

Saccol, A., Schlemmer, E., Barbosa, J. m-learning e u-learning - Novas perspectivas da aprendizagem móvel e ubíqua. São Paulo, SP: Pearson, 2010.

Sommerville, I. Engenharia de Software, 9a edição. São Paulo: Pearson Prentice Hall, 2011.

Vieira, F. J. R.and Nunes, M. A. S. N. DICA: Sistema de Recomendação de Objetos de Aprendizagem Baseado em Conteúdo, Scientia Plena, v. 8, 2012. 
XML. eXtensible Markup Language, Disponível em: <http://www.w3.org/XML/>. Acessado em: 30 Out. 2012.

Weiser, M. The computer for the 21 st century, Scientific America, v.265, no 3, p. 66-75, 1991.

WURFL. WURFL - Wireless Universal Resource FiLe, Disponível em: <http://wurfl.sourceforge.net/>. Acessado em: 25 Abr. 2012. 